\title{
Controlling microscopic friction through mechanical oscillations
}

\author{
R. Guerra, ${ }^{1}$ A. Vanossi, ${ }^{1}$ and M. Urbakh ${ }^{2}$ \\ ${ }^{1}$ CNR-INFM National Research Center S3 and Department of Physics, University of Modena and Reggio Emilia, \\ Via Campi 213/A, 41100 Modena, Italy \\ ${ }^{2}$ School of Chemistry, Tel Aviv University, 69978 Tel Aviv, Israel
}

(Received 14 May 2008; published 9 September 2008)

\begin{abstract}
We study in detail the recent suggestions by Tshiprut et al. [Phys. Rev. Lett. 95, 016101 (2005)] to tune tribological properties at the nanoscale by subjecting a substrate to periodic mechanical oscillations. We show that both in stick-slip and sliding regimes of motion friction can be tuned and reduced by controlling the frequency and amplitude of the imposed substrate lateral excitations. We demonstrate that the mechanisms of oscillation-induced reduction of friction are different for stick-slip and sliding dynamics. In the first regime the effect results from a giant enhancement of surface diffusion, while in the second regime it is due to the interplay between washboard and oscillation frequencies that leads to the occurrence of parametric resonances. Moreover, we show that for a particular set of parameters it is possible to sustain the motion with only the oscillations.
\end{abstract}

DOI: 10.1103/PhysRevE.78.036110

\section{INTRODUCTION}

In the emerging field of nanoscale science, major efforts have been devoted to form a deep, predictive understanding of the mechanisms involved in fundamental tribological processes, envisaging alternative innovative solutions to control friction [1]. The ability to manipulate frictional forces is extremely important for many technological applications. One may wish to reduce or enhance friction, eliminate the highdissipative chaotic and stick-slip regimes of motion, and instead, to achieve smooth sliding. Early stages of motion and stopping processes in micromechanical devices and computer disk drives, which exhibit chaotic stick-slip, pose, for example, a real problem. The difficulties in realizing an efficient control of friction are related to the complexity of the task, namely, dealing with systems with many degrees of freedom under a strict size interfacial confinement. The achievements of nanotechnology have opened new perspectives and new basic scientific questions in this direction, where novel local probes give access to the study of friction at the atomic level.

A novel approach for tuning frictional response, which has recently attracted considerable interest [2-8], is the mechanical control of a system, via externally imposed normal or lateral vibrations of small amplitude and energy to the sliding interface. Manipulations by mechanical excitations, when applied at suitable frequencies and amplitudes, can drive particles, which are close to the interface, out of their potential energy minima, thus increasing considerably surface diffusion and mobility, and reducing friction. Through the dynamical stabilization of desirable regimes of motion (e.g., smooth sliding), which would be otherwise unstable, substrate mechanical oscillations can reduce high dissipative sliding behaviors (stick-slip).

Since the idea is not to change the physical properties of mating interfaces, flexibility and accessibility are the main relevant features of this approach. Controlling frictional forces has been traditionally approached by chemical means $[9,10]$, usually by supplementing base lubricants with friction modifying additives. However, standard lubrication techniques provide prescribed tribological properties which
PACS number(s): 81.40.Pq, 68.35.Af, 62.25.-g, 05.40.-a

fit a certain range of parameters of the sliding objects (such as the load) and the external forcing, and are expected to be less effective at the nanoscales. Here, on the contrary, frictional properties can be tuned continuously by the frequency and the amplitude of the out-of-plane or in-plane vibrations of the sliding surfaces. The predicted effects should be amenable to atomic force microscopy (AFM) tests $[11,12]$ or applying ultrasound to the sample $[13,14]$, or even in studies of contact mechanics, of a probe interacting with oscillating quartz crystal microbalance surfaces [15-17].

In this paper, we extend the recent results [18] concerning the ability to modify tribological properties at the nanoscale by subjecting a substrate to lateral mechanical oscillations. The investigation is limited to a one-dimensional (1D) Tomlinson-type model. We show that microscopic friction can be suitably tuned and reduced by controlling the resonant frequencies and amplitudes of the imposed substrate lateral excitations. Depending on the model parameters, the numerical simulations reveal the details of the observed different regimes of motion (smooth sliding, stick-slip, inverted stickslip) and of the occurring transitions among them. Moreover, we show that for particular sets of parameter values the motion can be sustained just with the oscillations.

\section{MODEL AND NUMERICAL METHOD}

From a theoretical point of view, it has been frequently shown that, despite the simplified level of description, phenomenological models of friction have revealed the ability to mimick the main features of the tribological behavior observed both in nanoscale experiments or in more complex MD simulation frameworks [19].

The Prandtl-Tomlinson model is, probably, the most widely used in interpretation of tribological experiments due to its simplicity and its ability to account for the main physical features of atomic-scale friction (see, e.g., $[12,20]$ ). In this mathematical description, a particle of mass $m$ is coupled, via a harmonic spring of stiffness $K$, to a moving stage which slides at constant velocity $v_{G}$. Moreover, the particle experiences a periodic (sinusoidal) coupling $V(x)$ 
$=U \sin (2 \pi x / b)$ to the substrate, where $x$ is the current position of the particle. Introducing the viscous damping coefficient $\gamma$, which results from the energy dissipation to phonons or other excitations in the substrate, the equation of motion for the particle becomes

$$
m \ddot{x}+m \gamma \dot{x}=-\frac{d V(x)}{d x}-K\left(x-v_{G} t\right) .
$$

If the pulling spring is stiff enough (i.e., $K$ is larger than the effective substrate spring constant $\left.K_{0}=\max \left[V^{\prime \prime}(x)\right]\right)$, then there is always a unique equilibrium position $x_{\mathrm{eq}} \approx v_{G} t$ for the particle. Consequently, at small driving velocity, the friction will be linear in $v_{G}$. Things become more interesting once $K_{0}$ exceeds $K$. In this situation, the potential energy landscape experienced by the particle is characterized, at certain instances of time, by more than one stable position. The time dependence of the combined spring and substrate potential reveals that mechanically stable positions disappear at certain instances in time due to the motion of the spring. The system is locked in one of the minima of the potential energy landscape until the increasing elastic stress of the spring allows overcoming the barrier. After that, the slider is accelerated. The potential energy of the elastic stress is converted into the kinetic energy of the slip event and, eventually, dissipated into heat via the damping $\gamma$ term. Then, the particle rapidly drops to the next nearest metastable minimum, where it locks again. As a consequence, for sufficiently small driving velocity, the dissipated energy per sliding distance is rather independent of $v_{G}$, in agreement with many experimental results. Obviously, this simple mechanical description can provide only qualitative interpretations of the underlying tribological processes. The Tomlinson model is described in detail in many surveys, e.g., in Refs. [12,20], with a long list of applications to concrete physical systems (in particular, to those concerning tip-based devices).

In this work we consider a Tomlinson-type model as that recently introduced in Ref. [18]. In particular, in order to study the effect of lateral vibrations on $1 \mathrm{D}$ diffusion and friction, the model describes the diffusion and driven dynamics of an AFM tip (a single mass driven by an external guide) subject to a substrate, which oscillates in the lateral direction with amplitude $A$ and frequency $f$. The potential of such an oscillating substrate becomes

$$
V(x, t)=U \sin \left[\frac{2 \pi}{b}[x+A \sin (2 \pi f t)] .\right.
$$

The motion of the tip in lateral direction is governed by the equation

$$
m \ddot{x}=F_{s u b}(x, t)-K\left(x-x_{G}\right)+\Gamma(t)-m \gamma\left(\dot{x}-v_{s u b}\right),
$$

where $F_{s u b}$ is the force due to the substrate potential $V(x, t)$, and $x_{G}=v_{G} t ; v_{s u b}=-2 \pi f A \sin (2 \pi f t)$ represents the velocity at which the substrate oscillates. In particular, the last two terms take into account the thermal and dissipation effects in the framework of the Langevin dynamics [21]. The random force $\Gamma(t)$ and the viscous damping term $\gamma$ are related by the fluctuation-dissipation theorem as follows:

$$
\left\langle\Gamma(t) \Gamma\left(t^{\prime}\right)\right\rangle=2 \gamma m k_{B} T \delta\left(t-t^{\prime}\right),
$$

with $k_{B}$ and $T$ denoting the Boltzmann constant and the temperature, respectively. These two added terms can be thought of as representing degrees of freedom inherent in a real physical system which are not explicitly included in the adopted simplified mathematical description. In particular, we assume that the damping term in Eq. (3) is determined by the energy dissipation to phonons and electron-hole excitations in the substrate. As a result, this term is proportional to the relative velocity of the driven tip and the substrate. Under these conditions, the substrate vibrations cause a timeperiodic (ac) force acting on the tip, $F_{a c}$ $=m A(2 \pi f)^{2} \sin (2 \pi f t)$. This force presents the effect of inertia.

In the strong (weak) dissipative regime, when $\gamma$ is much larger (smaller) than the characteristic vibrational frequencies of the system, the motion is overdamped (underdamped). The numerical integration is carried out using the well-known velocity Verlet algorithm. For every value of the parameters and after reaching the steady state, the system characteristics of relevant physical interest are measured. Natural units used to characterize the model for length, time, and energy are $\AA, \mu s$, and meV. If not stated differently, $m$ $=1, U=100, b=1, K=12.63, T=0$, and $\gamma=32$ define our default parameter values. For parameters close to these default values, the system can be considered to behave as overdamped. Under these parameter ranges, the predicted effects, as for surface diffusion resonance frequencies, should be amenable, e.g., to AFM tests using the shear modulation mode, for which we typically have $m \approx 10^{-12}-10^{-14} \mathrm{~kg}, U$ $\simeq 0.25 \mathrm{eV}$, and $b \simeq 0.4 \mathrm{~nm}$. For these values of system parameters, the characteristic frequency turns out to be $f_{0}$ $=(1 / b) \sqrt{U / m} \approx 1-10 \mu \mathrm{s}^{-1}$ and the velocity unit is $\AA / \mu \mathrm{s}$.

\section{RESULTS AND DISCUSSION}

In order to investigate the effectiveness of lateral mechanical oscillations and their ability of improving nanoscale tribological properties, we have performed numerical simulations in different ranges of model parameters where the "natural" system (without oscillations) may exhibit both high dissipative stick-slip dynamics and smooth sliding behaviors.

At a fixed value of the amplitude $A(=1)$, Fig. 1 shows the influence of the substrate oscillation frequency $f$ on the timeaveraged friction force $\left\langle F_{L}\right\rangle=\left\langle K\left(x-x_{G}\right)\right\rangle$, for three distinct values of the driving velocity $v_{G}$, corresponding, respectively, to stick-slip dynamics for $v_{G}=1.6$ and to smooth sliding for $v_{G}=8$ and $v_{G}=16$. The frequency $f$ is varied adiabatically and, for each of its values, $F_{L}$ is averaged over a sufficiently long simulation time after reaching the dynamical steady state. Depending on the external stage velocity, the results presented in Fig. 1 exhibit two types of resonance minima of friction which are determined by different physical mechanisms. As we will show shortly, the low frequency resonance, which is most pronounced for the low driving velocity (e.g., $v_{G}=1.6$ ), results from the giant enhancement of surface diffusion induced by the oscillations of the substrate, as recently discussed in detail in Ref. [18]. On the 


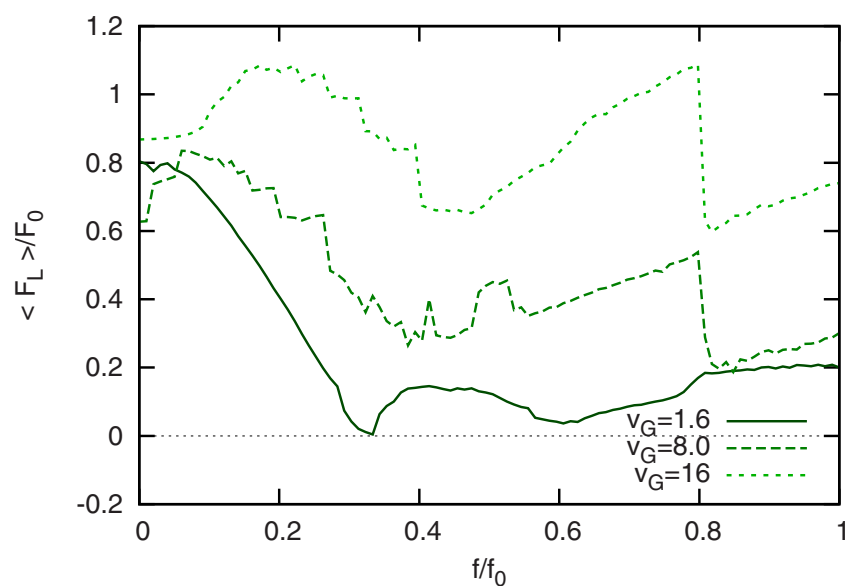

FIG. 1. (Color online) Averaged friction force dependence on the oscillation frequency externally imposed to the substrate. The behavior is shown for three different values of the driving stage velocity $v_{G}: 1.6$ (solid), 8.0 (dashed), and 16.0 (dotted). The lateral force $F_{L}=K\left(x-x_{G}\right)$ is normalized to the static friction force $F_{0}$ $=\max \left[V^{\prime}(x)\right]$, and the oscillation frequency $f$ to the system characteristic frequency $f_{0}=\frac{1}{b} \sqrt{\frac{U}{m}}$.

contrary, for higher driving velocities (e.g., $v_{G}=8.0 ; 16.0$ ), sharp resonance minima in the average lateral force $\left\langle F_{L}\right\rangle$ are caused by the interplay between the system washboard frequency $v_{G} / b$ and the oscillation frequency $f$.

An understanding of the effects of lateral substrate vibrations on the frictional properties of the system at low or moderate driving velocities, comes from the study of the diffusion coefficient for the undriven tip as a function of the oscillation frequency [18]. For low external frequencies $f$, the tip apex is able to follow the slow motion of the substrate, performing just small vibrations around the potential minima. Under this condition, if the thermal energy is sufficiently smaller than the height of the potential barrier, the probability to escape from the potential well is exponentially small. For the optimal matching of the oscillation frequency $f$ and the amplitude $A$, the diffusivity $D$ may be considerably enhanced [22]. In this case, the tip approaches the maximum of the substrate potential at the end of the half cycle of the surface oscillations, where the driving force acting on the tip diminishes. Then, even a weak thermal noise splits the ensemble of tips into two parts that relax to the neighboring minima of the surface potential, and the resonance enhancement of diffusion is observed. A further increase of the frequency leads again to localized dynamics; in contrast to the case of low frequencies here the tip overcomes the potential barriers and oscillates between neighboring minima of the surface potential. The resonance enhancement of surface diffusion leads to a corresponding reduction of the mean friction force at $f \simeq 3.32$ which is most pronounced for low driving velocities (see Fig. 1 for $v_{G}=1.6$ ). We found that the curves of Fig. 2 calculated for three oscillation amplitudes $A$ are not changed qualitatively with an increase of temperature; the diffusion resonance peaks are still visible for higher temperatures but are greatly broadened and damped. The driving force acting on the tip is proportional to the amplitude of oscillations and, as a result, we observe that the resonance frequency decreases with $A$.

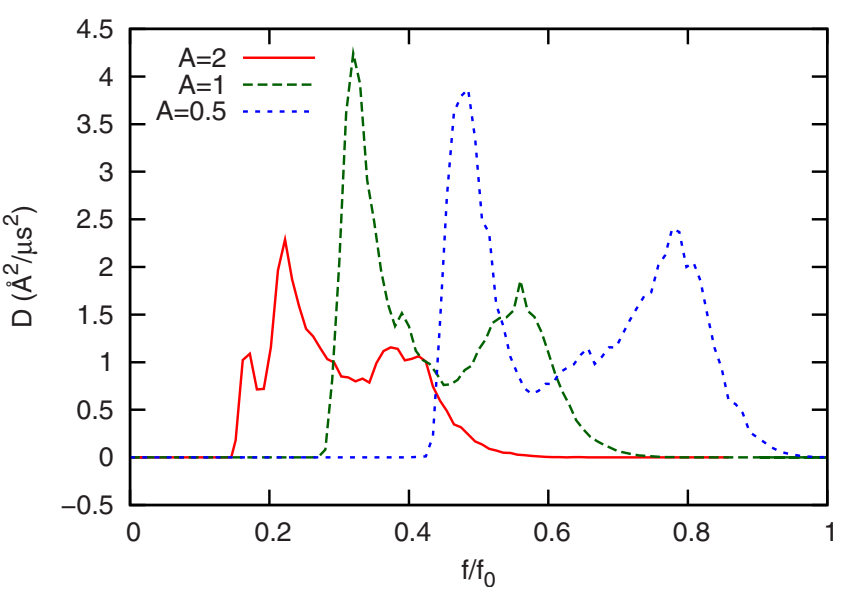

FIG. 2. (Color online) Diffusion $D$ of the tip as a function of the normalized oscillation frequency $f / f_{0}, f_{0}=\frac{1}{b} \sqrt{\frac{U}{m}}$, at finite temperature $\left(k_{B} T / U=0.01\right)$, for three different values of the oscillation amplitude $A$.

By systematically scanning the phase space of the model parameters, it is possible to tune the characteristics of the external oscillating excitations, in terms of suitable choices of amplitude and frequency, to achieve very favorable sliding conditions, for which friction almost vanishes or even $F_{L}$ $\leqslant 0$ on average. For a negative value of the lateral friction force, the driving guide, moving at speed $v_{G}$, does not pull the tip apex anymore, but it is instead pushed forward by the tip itself. An example of such a sliding situation is shown in Fig. 3, where, once the external guide moving at $v_{G}=1.6$ is detached from the tip, the direct tip motion is fully sustained by the only sinusoidal oscillations of the substrate, at the diffusion resonance $f=3.32$. Due to highly nonlinear and strongly fluctuating dynamics, the determination of a desirable set of model parameters achieving this "negative" friction regime is not always a trivial task.

For higher driving velocities, such as those shown in Fig. 1 (i.e., $v_{G}=8.0 ; 16.0$ ), the mechanism of reduction of friction

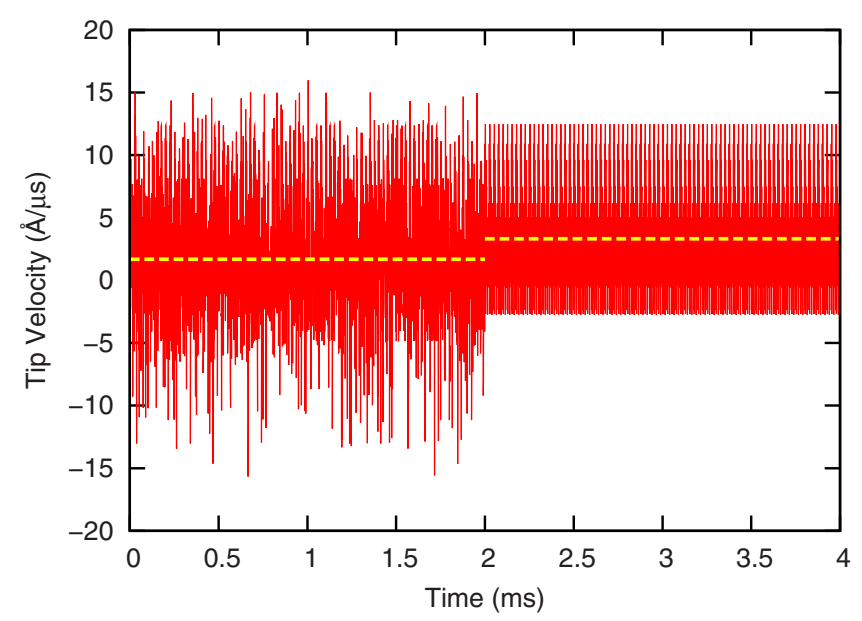

FIG. 3. (Color online) During the first $2 \mathrm{~ms}$ the tip is driven at $v_{G}=1.6$ and then detached from the guide. The substrate oscillates uninterruptedly over the whole simulation with $f=3.32$ and $A$ $=1.44$. Clearly, the tip motion is completely carried by the substrate vibrations, with an average velocity highlighted by the dashed line. 


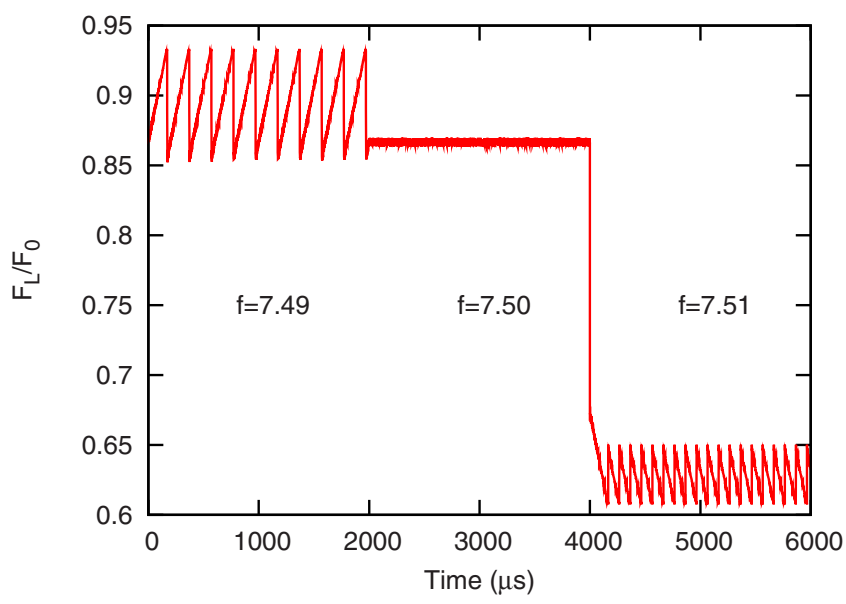

FIG. 4. (Color online) $F_{L} / F_{0}$ time-dependence passing through a resonance frequency. At $A=1.44$ and $v_{G}=15$, the oscillation frequency is increased from $f=7.49(0-2000 \mu \mathrm{s})$ to $f=7.50=v_{G} / 2$ $(2000-4000 \mu \mathrm{s})$, up to $f=7.51(4000-6000 \mu \mathrm{s})$. The lateral force oscillations are almost completely washed out at the resonance frequency. The system shows, across the resonance, a transition from a stick-slip-like to an inverted stick-slip-like regime.

is essentially different, stemming from the interplay between the system washboard frequency $v_{G} / b$ and the oscillation frequency $f$. This resonant behavior of the driven tip is clearly displayed by sudden and sharp drops of $\left\langle F_{L}\right\rangle$ at specific oscillation frequencies $f_{R}$, which are given by the formula $f_{R}=v_{G} / N b$, with $N$ integer.

For an in-depth investigation of these features, we have analyzed the detailed dynamics during the transition across a resonance frequency $f_{R}=v_{G} / 2 b$ for the case of an external guide velocity $v_{G}=15$ (see Fig. 4). Due to a dynamical combination of fast sliding and substrate oscillations, for frequencies $f \lesssim f_{R}$ the lateral force time series shows an asymmetric sawtooth shape resembling that of the usual stick-slip but with very tiny amplitude; as soon as $f=f_{R}$ the lateral force stops to oscillate, meaning that the guide-tip separation remains constant. Finally, when $f \gtrsim f_{R}$ the synchronization is lost and $F_{L}$ starts again oscillating with doubled frequency, but showing an inverted stick-slip-like behavior. The resonance marks in general the transition from a negative to a positive phase difference between the characteristic washboard frequency $v_{G} / b$ and the oscillation frequency $f$, which is characteristic for parametric resonances. This effect can lead to a significant reduction of friction in the sliding regime of motion, as shown by sharp drops in average and instantaneous lateral forces presented in Figs. 1 and 4 . It should be noted that this mechanism of reduction of friction in the sliding state is very different for that discussed above for the stick-slip regime.

During the adiabatic increasing procedure of $f$, it has been noted that switching to the resonance $f_{R}$ at different times may result in distinct values of lateral friction force because of the different initial conditions. Due to commensuration between the washboard $v_{G} / b$ and the frequency $f$ at the resonance $f_{R}$, this behavior shows in Fig. 5 the occurrence of only a discrete set of allowed values for $\left\langle F_{L}\right\rangle$; the even separation between these states is determined by the dimensionless constant $K b / F_{0}$.

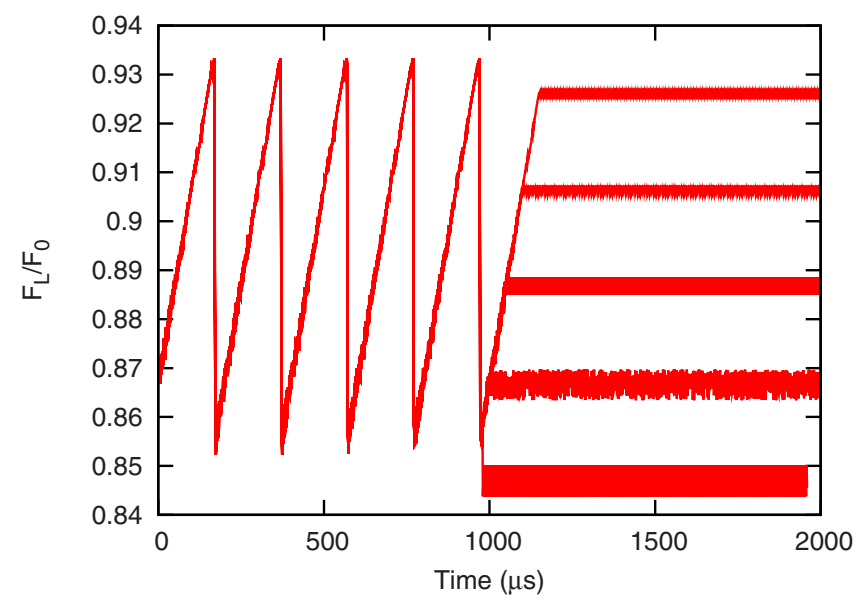

FIG. 5. (Color online) Lateral force vs time in approaching the resonance frequency $f=7.50=v_{G} / 2$. Switching to the resonance at different times may result in a discrete set of distinct and evenly separated values of $F_{L}$ due to different initial conditions.

\section{CONCLUSIONS}

In summary, the model demonstrates the effectiveness of imposed lateral excitations for the reduction and control of friction at the nanoscale under different regimes of motion (from stick-slip to smooth sliding) of a mating contact. For an optimal matching of the oscillation frequency and amplitude, this mechanical manipulation may drive, for example, an interfacial system out of its potential energy minima, thus increasing considerably surface diffusion and mobility, and reducing friction. Unfavorable high dissipative stick-slip motions can be suppressed in favor of the dynamical stabilization of desirable smooth-sliding regimes.

It has been shown that physical mechanisms of the oscillation-induced reduction of friction are different for stick-slip and sliding regimes of motion. For stick-slip dynamics, at low driving speed, giant enhancement of surface diffusion takes place. In this case, the observed tribological response may turn to be quite sensitive to thermal effects, able to spoil the sharp resonant nature of this phenomenology. Differently, for smooth-sliding regimes at high driving velocity, it is the interplay between washboard and oscillation frequencies that determines the occurrence of favorable parametric resonances. A very careful tuning of the model parameters has been proved to lead to the possibility of a directed motion sustained by the sinusoidal substrate oscillations only.

\section{ACKNOWLEDGMENTS}

We are grateful to Alexander E. Filippov for invaluable discussions. This research was partially supported by PRRIITT (Regione Emilia Romagna), Net-Lab "Surfaces \& Coatings for Advanced Mechanics and Nanomechanics" (SUP\&RMAN). A.V. thanks the School of Chemistry at Tel Aviv University for the kind hospitality and the ESF "Nanotribology" Program for financial support. M.U. acknowledges the support by the Israel Science Foundation (Grant No. 1116/05). 
[1] M. Urbakh, J. Klafter, D. Gourdon, and J. N. Israelachvili, Nature (London) 430, 525 (2004).

[2] M. G. Rozman, M. Urbakh, and J. Klafter, Phys. Rev. E 57, 7340 (1998).

[3] J. P. Gao, W. D. Luedtke, and U. Landman, J. Phys. Chem. B 102, 5033 (1998).

[4] A. Socoliuc, E. Gnecco, S. Maier, O. Pfeiffer, A. Baratoff, R. Bennewitz, and E. Meyer, Science 313, 207 (2006).

[5] M. Heuberger, C. Drummond, and J. N. Israelachvili, J. Phys. Chem. B 102, 5038 (1998).

[6] V. Zaloj, M. Urbakh, and J. Klafter, Phys. Rev. Lett. 82, 4823 (1999).

[7] A. Cochard, L. Bureau, and T. Baumberger, J. Appl. Mech. 70, 220 (2003).

[8] M. Igarashi, J. Nakamura, and A. Natori, Jpn. J. Appl. Phys., Part 1 46, 5591 (2007).

[9] O. Dudko, A. E. Filippov, J. Klafter, and M. Urbakh, Tribol. Lett. 12, 217 (2002).

[10] N. J. Mosey, M. H. Müser, and T. K. Woo, Science 305, 1612 (2005).

[11] E. Riedo, E. Gnecco, R. Bennewitz, E. Meyer, and H. Brune, Phys. Rev. Lett. 91, 084502 (2003).

[12] M. H. Müser, M. Urbakh, and M. O. Robbins, Adv. Chem.
Phys. 126, 187 (2003).

[13] S. Ge, Y. Pu, W. Zhang, M. Rafailovich, J. Sokolov, C. Buenviaje, R. Buckmaster, and R. M. Overney, Phys. Rev. Lett. 85, 2340 (2000).

[14] F. Dinelli, S. K. Biswas, G. A. D. Briggs, and O. V. Kolosov, Appl. Phys. Lett. 71, 1177 (1997).

[15] B. Borovsky, J. Krim, S. A. Syed Asif, and K. J. Wahl, J. Appl. Phys. 90, 6391 (2001).

[16] S. Berg and D. Johannsmann, Phys. Rev. Lett. 91, 145505 (2003).

[17] J. F. Lubben and D. Johannsmann, Langmuir 20, 3698 (2004).

[18] Z. Tshiprut, A. E. Filippov, and M. Urbakh, Phys. Rev. Lett. 95, 016101 (2005).

[19] A. Vanossi and O. M. Braun, J. Phys.: Condens. Matter 19, 305017 (2007).

[20] M. O. Robbins, in Jamming, Friction and Unsteady Rheology, edited by A. J. Liu and S. R. Nagel, Jamming and Rheology: Constrained Dynamics on Microscopic and Macroscopic Scales (Taylor and Francis, London, 2000).

[21] H. Risken, The Fokker-Planck Equation (Springer, Berlin, 1984).

[22] M. Schreier, P. Reimann, P. Hanggi, and E. Pollak, Europhys. Lett. 44, 416 (1998). 